\title{
Abnormalities of lipid profile in overweight and obese Indian children
}

\author{
Minakshi B ${ }^{1}$, Chithambaram N S \\ ${ }^{1}$ Dr.Minakshi B, Department of Paediatrics, VIMS \& RC, Bangalore, ${ }^{2}$ Dr.Chithambaram N S, Professor, Department of \\ Paediatrics, VIMS \& RC, Bangalore, India.
}

Address for correspondence: Dr. N S Chithambaram, Email:chithams1@ gmail.com

\begin{abstract}
Objective: To identify the abnormalities of lipid profile early in overweight and obese children. Study design: Observational study. Setting: Vydehi Institute of Medical Sciences \& Research Centre, Bangalore. Participants: 100 children who are overweight and obese. Outcome Measures: Abnormal lipid profile. Methods: In all patients who are overweight and obese as per IAP growth charts and who are above 6 years of age, a detailed history including antenatal history, birth weight, diet history, personal history were taken. In these patients demographic measures like age, sex, anthropometric measurements weight, height, BMI, waist circumference, waist hip ratio and blood pressure were recorded. In all the patients fasting blood glucose, triglyceride and HDL-C were done. Results: Out of 100 children studied, 61\% (61cases) were overweight and 39\% (39 cases) were obese. Males were 52\% (52 cases) and females were $48 \%$ (48 cases). 92 cases had normal birth weight, 6 cases were SGA and 2 cases were LGA babies. 85\% had TGL levels less than $150 \mathrm{mg} / \mathrm{dl}$ and $15 \%$ had high or equal to $150 \mathrm{mg} / \mathrm{dl} .69 \%$ of the cases had HDL-C less than $40 \mathrm{mg} / \mathrm{dl}$ and $31 \%$ of the cases had HDL-C more than or equal to $40 \mathrm{mg} / \mathrm{dl}$. Conclusions: By doing lipid profile in overweight and obese children, we can identify abnormal lipid profile in these children early and initiate non pharmacological management, behavioral therapy and if required pharmacological therapy thereby preventing these children from developing metabolic syndrome and its associated long term complications.
\end{abstract}

Keywords: Lipid profile; Overweight; Obesity.

\section{Introduction}

Childhood obesity appears with a powerful array of cardiovascular risk factors including combined dyslipidaemia, insulin resistance and hypertension and has been shown to be associated with pathologic evidence of accelerated atherosclerosis in autopsy studies.

The dyslipidaemia pattern associated with childhood obesity consists of a combination of elevated triglycerides (TG), decreased high density lipoprotein cholesterol (HDL-C), and top normal to mildly elevated low density lipoprotein cholesterol (LDL-C). Recent NHANES data indicate this pattern is highly prevalent, present in $42.9 \%$ of children with $\mathrm{BMI}>95^{\text {th }}$ centile [1].

Manuscript received: $14^{\text {th }}$ July 2016

Reviewed: $28^{\text {th }}$ July 2016

Author Corrected; $7^{\text {th }}$ August 2016

Accepted for Publication: $19^{\text {th }}$ August 2016
Along with overweight or obesity, if there are abnormalities of lipids such elevated TG, decreased HDL C, impaired glucose and hypertension, then we call this as Metabolic Syndrome which has long term implications on the child as it grows older.

Hence it is necessary to identify the above abnormalities in all overweight or obesity children at the earliest and initiate appropriate specific treatment to prevent progression to Metabolic Syndrome and prevent its complications.

India, a developing country is already known as Diabetic Capital, has a large number of children with overweight and obesity which further add to the problem [2]. Hence this study was done to detect the 
abnormalities of lipid profile early in overweight and obese Indian childrenabove 6 years of age.

\section{Materials and Methods}

This observational study was conducted at Vydehi Institute of Medical Sciences and Research Centre, Bangalore between December 2013 to January 2015, where 100 Indian children above 6 years of age who were overweight and obese were included in the study.

All patients above 6 years of age who are overweight and obese are enrolled in the study. Children with known diabetes, on long term medications which cause obesity, syndromic conditions with obesity and endocrine disorders which cause obesity were excluded from the study. A detailed history including antenatal history for gestational diabetes mellitus, birth weight for IUGR, diet history, and personal history was taken. In these patients demographic measures like age, sex, anthropometric measurements like weight, height, BMI, waist circumference, waist hip ratio and blood pressure were recorded. In all patients, Fasting blood glucose, triglyceride, and HDL-C were done.

Body weight was measured to the nearest $0.1 \mathrm{~kg}$ with a balance scale (Baurer, PS 07), and height was measured to the nearest $0.1 \mathrm{~cm}$ with stadiometer (Hyssna Limfog, $\mathrm{AB})$ with subjects lightly dressed and without shoes. Body mass index (BMI) was calculated as weight $(\mathrm{kg})$ divided by height square $(\mathrm{m} 2)$.

Fasting blood glucose and TG measurements were performed using enzymatic assays (Instrumentation Lab, MA, USA). HDL-C was measured by a direct enzymatic assay without precipitation (Instrumentation Lab, MA, USA).

Serum TG and HDL-C were considered high or low when they fell above or below the recommended values. Data were analyzed using STATA for Windows version 10.1 (Stata Corp, College Station, TX,USA). The chi square test or the Fisher's exact test were done to analyze the data.

\section{Results}

Out of 100 children studied, 61\% (61cases) were overweight and 39\% (39 cases) were obese. Males were $52 \%$ (52 cases) and females were $48 \%$ (48 cases). 92 cases had normal birth weight, 6 cases were SGA and 2 cases were LGA babies. Consumption of junk food, one of the risk factors for the weight gain was noted in $95 \%$ of the cases studied in the present study. All the cases undertaken had 4-6 hours of screen time which includes television, video games, higher gadgets and mobile phones. Lack of physical activity was noted in all the cases. (Table 1).

Table 1: Demographic profile of subjects.

\begin{tabular}{|c|c|c|c|c|}
\hline & \multicolumn{3}{|c|}{$n=100$} & \\
\hline No & Variables & & & \\
\hline 1 & Gender & Male $52(52 \%)$ & Female $48(48 \%)$ & \\
\hline 2 & Birth weight & AGA $92(92 \%)$ & SGA $6(6 \%)$ & LGA $2(2 \%)$ \\
\hline 3 & Junk food consumption & Yes $100(100 \%)$ & --- & \\
\hline 4 & Screen Time & $>4-6$ hrs $100(100 \%)$ & $\begin{array}{c}-- \\
\end{array}$ & \\
\hline 5 & Physical activity & Minimal $100(100 \%)$ & --- & \\
\hline
\end{tabular}

$85 \%$ had TGL levels less than $150 \mathrm{mg} / \mathrm{dl}$ and $15 \%$ had high or equal to $150 \mathrm{mg} / \mathrm{dl} .69 \%$ of the cases had HDL-C less than $40 \mathrm{mg} / \mathrm{dl}$ and $31 \%$ of the cases had HDL-C more than or equal to $40 \mathrm{mg} / \mathrm{dl}$. (Table 2)

Table 2: Lipid profile of children.

\begin{tabular}{|c|c|c|c|}
\hline \multicolumn{2}{|c|}{ Trigylcerides } & \multicolumn{2}{c|}{ HDL - C } \\
\hline$<150 \mathrm{mg} / \mathrm{dl}$ & $>150 \mathrm{mg} / \mathrm{dl}$ & $<40 \mathrm{mg} / \mathrm{dl}$ & $>40 \mathrm{mg} / \mathrm{dl}$ \\
\hline $85(85 \%)$ & $15(15 \%)$ & $69(69 \%)$ & $31(31 \%)$ \\
\hline
\end{tabular}




\section{Discussion}

Childhood obesity appears with a powerful array of cardiovascular risk factors including combined dyslipidemia, insulin resistance and hypertension and has been shown to be associated with pathologic evidence of accelerated atherosclerosis in autopsy studies [3].

The dyslipidemia pattern associated with childhood obesity consists of a combination of elevated triglycerides (TG), decreased high density lipoprotein cholesterol (HDL-C), and top normal to mildly elevated low density lipoprotein cholesterol (LDL-C).

Normal TG levels are $<100 \mathrm{mg} / \mathrm{dL}$ in children younger than age 10 years and $<130 \mathrm{mg} / \mathrm{dL}$ at ages $10-18$ years [3]. In the dyslipidemia associated with obesity, TG levels are usually between 100 and 400 mg/dL. Recent NHANES data indicate this pattern is highly prevalent, present in $42.9 \%$ of children with BMI $>95^{\text {th }}$ centile [1].

Insulin resistance, another common feature in obese children and adolescents, contributes significantly to development of the combined dyslipidemia of obesity by enhancing hepatic delivery of non-esterified free fatty acids for triglyceride (TGL) production and sequestration into triglyceride-rich lipoproteins.

High TGL levels are processed into small, dense LDL and small, less stable HDL; there is both an increase in small, dense LDL and in overall LDL particle number and a reduction in total HDL-C and in large HDL particles with analysis by nuclear magnetic resonance spectroscopy (NMR). The combined dyslipidemia pattern seen with traditional lipid profile analysis identifies the atherogenic pattern seen with NMR analysis [4].

The combined dyslipidemia of obesity is particularly atherogenic for multiple reasons: small dense LDL particles are inefficiently cleared by LDL receptors, elevated total circulating LDL particles heighten the risk of entrapment in the sub endothelial matrix, and decreased large HDL particles limit reverse cholesterol transport. The atherogenicity of the combined dyslipidemia seen with childhood obesity manifests in structural and functional vascular changes assessed non- invasively as increased carotid intima-media thickness (cIMT) and increased arterial stiffness. In children, a recent report from the longitudinal Young Finns study revealed that, at 21-year follow-up, subjects with the combined dyslipidemia pattern beginning in childhood had significantly increased cIMT compared with normolipidemic controls, even after adjustment for other risk factors.

A paper from the CDC evaluated multiple CVD risk factors in US children, specifically, showed that the combined dyslipidemia pattern seen with obesity in childhood is increasing in prevalence and predicts vascular dysfunction in young adulthood and early clinical events in adult life $[5,6]$.

The role of TG has been controversial for decades and even if more recent epidemiologic studies demonstrate that plasma TG levels predict cardiovascular disease, their role is still questionable [7]. Increasing TG levels cause profound changes in the physicochemical composition of HDL, VLDL, and LDL particles, and the particle core, represented by cholesterol esters, is progressively depleted and replaced by TG.

Dyslipidemia in children and adolescents has become a frequent clinical condition, especially due to the increase in overweight and obesity prevalence in this age range [8].

Gilles Plourde studied Impact of obesity on glucose and lipid profiles in adolescents at different age groups in relation to adulthood from 1974 to 2000. This retrospective-prospective longitudinal study confirmed that adolescents aged between 13 and 15 years old of both sexes with a BMI $\geq 85^{\text {th }}$ percentile are at increased risk of becoming overweight or obese adults and presenting abnormal glucose and lipid profiles as adults. This emphasizes the importance of early detection and intervention directed at treatment of obesity to avert the long-term consequences of obesity on the development of cardiovascular diseases [9].

In another study by Anoop Misra and Naval K Vikram in 2004, there was high prevalenc of excess body fat, adverse body fat patterning, hypertriglyceridemia, and insulin resistance beginning at a young age irrespective of their geographic locations. These data suggest that 
primary prevention strategies should be initiated early in this ethnic group [10].

The $2005,1^{\text {st }}$ Guideline for Atherosclerosis Prevention in Children and Adolescents recommends that every child over 10 years old assesses their cholesterol levels [11]. Such recommendations are more important when children present any of the risk factors such as family history of early atherosclerosis in parents, siblings or grandparents (before 55 years old in men and before 65 years old in women), if parents have cholesterol levels $>240 \mathrm{mg} / \mathrm{dl}$; if children have other associated risk factors, such as hypertension, obesity and saturated fat or trans fatty acid rich diet, Children with associated with dyslipidaemia, such as AIDS and hypothyroidism, who use drugs which may predispose them to dyslipidaemia, e.g., isotretinoin for acne and children who present clinical manifestations of dyslipidaemia, even if they are not pathognomonic, such as xanthoma and/or xanthelasma [11].

Treatment depends on the type of dyslipidaemia, i.e., if the cause is familial, with clinical signals such as xanthomas or if dyslipidaemia is inserted in the global picture of metabolic syndrome.

The lipid profile is completely diverse: while in familial dyslipidaemia there is significant hypercholesterolemia with LDL-c levels possibly over $400 \mathrm{mg} / \mathrm{dl}$ in dyslipidaemia associated with metabolic syndrome, there is a higher trend of hypertriglyceridemia, low HDL-c levels, and LDL-c levels without quantitative but rather qualitative changes, such as small dense particles which are more atherogenic.

Dyslipidaemia at this age range may have a genetic component with or without the environmental component, such as improper diet, familial hypercholesterolemia, familial combined hyper-lipidaemia and hypertriglyceridemia and there may be types of dyslipidaemia which are predominantly environmentrelated, but which may also have a genetic component [12].

A complex dyslipidemia, which is an integral part of the underlying insulin resistance in Type 2 diabetes mellitus group, is a key to this increased risk. Increased secretion of VLDL from the liver is a central feature of dyslipidemia and is linked significantly to the low HDL and abnormal LDL that are also present.

A number of physiologic and pharmacologic approaches are available and should be used aggressively to treat diabetic dyslipidemia $[13,14]$.

The prognosis depends on the type of dyslipidaemia, and it is well established that hypercholesterolemia, in its homozygotic form, is extremely severe with a prevalence of 1 in 1 million.

Male children usually of 15 years of age may already present a coronary event or death. For dyslipidaemia associated with metabolic syndrome, recent data have shown that the presence of metabolic syndrome during childhood is associated with the development of coronary disease 25 years later, i.e., in adulthood.

\section{Conclusion}

Detection of abnormalities in lipid profile such as high TG and low HDL-C levels in overweight and obese children can help in preventing the child from progression to metabolic syndrome.

Moreover we should initiate appropriate non pharmacologic or pharmacologic treatment early to prevent the complications of abnormal lipids.

Funding: Nil, Conflict of interest: Nil

Permission from IRB: Yes

\section{References}

1.Alberti KG, Eckel RH, Grundy $\mathrm{SM}$, et al. Harmonizing the metabolic syndrome: a joint interim statement of the International Diabetes Federation Task Force on Epidemiology and Prevention; National Heart, Lung, and Blood Institute; American Heart Association; World Heart Federation; International Atherosclerosis Society; and International Association for the Study of Obesity.Circulation. 2009 Oct 20;120(16):1640-5. doi: 10.1161/Circulation AHA.109.192644.Epub 2009Oct 5.

2. S. Goenka, D. Prabhakaran, V. S. Ajay, and K. S. Reddy, "Preventing cardiovascular disease in IndiaTranslating evidence to action," Current Science. 2009; 97(3):367-377. 
3. Stephen Cook, Rae Ellen W. Kavey, Dyslipidemia and Pediatric Obesity.Pediatr Clin North Am. 2011 Dec; 58(6):1363-1373.doi: 10.1016/j.pcl. 2011. 09.003.

4. Laakso M, Sarlund H, Mykkänen L. Insulin resistance is associated with lipid and lipoprotein abnormalities in subjects with varying degrees of glucose tolerance.Arteriosclerosis. 1990 Mar-Apr; 10(2):223-31.

5.Justin P. Zachariah. Improving Blood Pressure in Children Is Protective Over the Long Term. Circulation. 2013;128:198-9. DOI: 10.1161/Circulation AHA.113. 003954 .

6. N. Mattsson, T. Rönnemaa, M. Juonala,J. S. A. Viikari and O. T. Raitakari.The prevalence of the metabolic syndrome in young adults. The Cardiovascular Risk in Young Finns Study.Journal of Internal Medicine.2007;261,: 159-69.doi: 10.1111/j. 1365-2796.2006.01752.x.

7. Adeli K., Taghibiglou C., van Iderstine S. C., Lewis G. F. Mechanisms of hepatic very low-density lipoprotein overproduction in insulin resistance. Trends in Cardiovascular Medicine. 2001;11(5):170-176. doi: 10.1016/s1050-1738(01)00084-6.

8. Rio-Navarro BE, Velazquez-Monroy O, SanchezCastillo CP, et al. The high prevalence of overweight and obesity in Mexican children.Obes Res. 2004; $12: 215-23$

9. Gilles Plourde.Impact of obesity on glucose and lipid profiles in adolescents at different age groups in relation to adulthood.BMC FamPract.2002; 3: 18.doi: 10.1186/ 1471-2296-3-18.

10. Anoop Misra,Naval K VikramInsulin resistance syndrome (metabolic syndrome) and obesity in Asian Indians: evidence and implications.2004;20(5):482-91 .DOI: http://dx.doi.org/10.1016/j.nut.2004.01.020.

11. Back GI, Caramelli B, Pellanda L, Duncan B, Mattos S, Fonseca FH. I Guidelines of Prevention of Atherosclerosis in Childhood and Adolescence. Arq Bras Cardiol. 2005;85(Suppl 6):4-36.

12. Alfredo Halpern, Marcio C Mancini, Maria Eliane C Magalhães,et al.Metabolic syndrome, dyslipidemia, hypertension and type 2 diabetes in youth: from diagnosis to treatment.Diabetol MetabSyndr.2010;2: 55. 13. Chahil TJ, Ginsberg HN.Diabetic dyslipidemia. Endocrinol Metab Clin North Am. 2006; 35(3):491510, vii-viii.

14. Ginsberg, H. N., Zhang, Y.-L. and Hernandez-Ono, A. (2006), Metabolic Syndrome: Focus on Dyslipidemia. Obesity, 14: 41S-49S. doi: 10.1038/oby. 2006.281 .

\section{How to cite this article?}

Minakshi B, Chithambaram N S. Abnormalities of lipid profile in overweight and obese Indian children. Int J Pediatr Res.2016;3(8):580-584.doi:10.17511/ijpr.2016.i08.06 